\section{NOTE ON THE TREATMENT OF DIPHTHERIA.}

By JAMES SAWYER, M.D.LOND., M.R.C.P., Physician to the Queen's Hospital, Birmingham.

So far as I have observed, the value of the local application to the diseased mucous surfaces of remedies which have the power of dissolving diphtheritic membrane has often been overlooked, and has only exceptionally been recognised, in the numerous disquisitions on the therapeutics of diphtheria which have lately flooded our medical periodicals. Lactic acid and lime-water will each dissolve diphtheritic membrane; their solvent power has been proved clinically, and it has been shown that pieces of detached membrane can be dissolved in a test-tube by either of these agents. I have successfully employed lactic acid and lime-water in the cases of diphtheria I have treated during the last two years. Early in 1877 , I saw a young lady suffering from pharyngeal diphtheria, in consultation with Dr. Crane of Hallaton, Leicestershire. In this case, the most marked benefit followed the use of a spray of solution of lactic acid directed, at intervals of two hours, upon the affected surfaces. I shortly afterwards treated the wife of a physician for pharyngeal diphtheria. In this case, I advised the use of sprays of lime-water and lactic acid alternately, at intervals of one hour. The good eftect of these remedies was very decided; my patient was especially grateful for the great comfort she experienced from the soothing local action of the spray of lime-water. I regard the employment of these topical solvents as of imperative importance. I do not think we can justly omit their use in any case of membranous diphtheria. They should, of course, supplement, and not supplant, the well known constitutional and general treatment of an affection which is constitutional and general in many of its most prominent features. Even when the larynx is involved, the spray may possibly still be useful, for the solvent particles may be drawn to the part during inspiration. I use Corbyn's excellent throat-spray, the officinal limewater, and an aqueous solution of lactic acid, of the strength of fifteen to twenty grains of the acid to an ounce of water. In the admirable report on the lamentable outbreak of diphtheria in the Grand Ducal family of Hesse-Darmstadt, published in the JourNad, Dr. Oertel remarks : "Repeated experimental investigations and bedside observations lead me to the belief that lime-water is still the best means for the solution of fibrinous membranes." In the valuable monograph on diphtheria, just published, from the pen of my friend Dr. Morell Mackenzie, I find the following testimony to the efficacy of the local use of the solvents I have mentioned : "The chief of these (local agents which act as solvents) are : lime-water, solution of caustic potash, chlorate of potash, and lactic acid. Added to pieces of detached membrane in a test-tube, each of these substances has certainly the power of dissolving them ; and whilst the false membrane is in contact with the living tissues, they have a similar, though less active, effect."

\section{CLINICAL MEMORANDA.}

\section{SPREADING QUINSY.}

THE admirable paper of Dr. Cornelius Fox, read before the last meeting of the British Medical Association and published in the BRITISH MEDICAL Journal of December 7 th, places before the profession in a clear light the symptoms of a throat-affection, which he most aptly terms "spreading quinsy".

Early last spring, I attended a large number of patients suffering from this complaint. In a district a few miles off, there was a pretty severe epidemic of diphtheria at the same time, and consequently there was much anxiety lest we should have an outbreak of this most dangerous disease. I carefully examined each case that came under my notice, and I was convinced that not one of them could be considered to be true diphtheria ; and, being at a loss for a term to describe the affection, I named it in my note-book "epidemic quinsy". In each case, I found that there was some sanitary defect in the residence or in the immediate neighbourhood of the patient, proving conclusively to my mind that this belongs to that class of disorders termed filth-diseases.

At the commencement, the patient complains of lassitude, pains in the limbs, slight shiverings, and disordered stomach. Some marked pyrexia sets in, and, at the same time, pricking sensations in the throat, with an attendant propensity to constant hawking, are complained of. On examination of the throat, the tonsils are seen to be enlarged and inflamed, the redness extending over the pharynx and fauces, and involving the uvula. Yellowish opaque spots, and sometimes even large patches, subsequently appear on the tonsils. These can be easily detached, and probably consist only of the accumulated secretions from the follicles. There is much tenderness under the jaw, and the salivary and adjacent lymphatic glands are generally swollen and painful. I have observed that, in these cases, the tonsils do not become enlarged to anything like the extent usually met with in a severe attack of ordinary cynanche tonsillaris, and that they are of a deeper and more livid hue, and a more glazed and shining appearance. The pulse soon exceeds 100 , and the temperature generally rises to about IO2 deg.; in one case, it reached as high as 103.8 deg. Towards evening, there is occasionally slight delirium, and almost invariably great restlessness. The urine in no case contains any trace of albumen; it is distinctly febrile, and there is a marked deficiency of the chlorides. The patient soon becomes very weak and low, and the difficulty in swallowing tends still further to increase the prostration. The case is generally at the worst on the fifth or sixth day, after which the patient gradually commences to improve; there is no crisis, and, as a rule, the tonsils do not suppurate. I have never noticed sequelæ of any kind, with the exception perhaps of slight weakness of the throat, and an increased tendency to take cold.

As to treatment, I found it best, at the outset of the case, to administer a saline aperient, and then, for the first two or three days, to give a saline mixture. Subsequently, tonics, and especially quinine, are not only serviceable, but in most cases even necessary. When the patient becomes feverish, restless, and feeble, I have found a moderate quantity of wine decidedly beneficial. Milk and beef-tea form the best food throughout, as they are comparatively easy to swallow, and, of course, highly nutritious. A warm atmosphere is the best, and frequent irrigation of the throat with warm water is productive of considerable benefit. Where the patches have been unusually adherent, and the inflammation more than ordinarily severe, I have painted the tonsils with tincture of steel and glycerine. Within a fortnight, the patient is almost invariably convalescent.

Fred. H. Varley Grosholz, L.K.Q.C.P.I., Aberdovey.

\section{OBSTETRIC MEMORANDA.}

\section{RUPTURE OF VAGINA DURING PARTURITION, FOL- LOWED BY DEATH IN NINE HOURS.}

ON November 2oth, A. H., aged 27, a stout woman, was delivered of her first child. Labour had fairly set in about six hours previously; but the pains were not severe till 2 A.M.; the child, a full-sized male, being born at 3 o'clock. The birth took place immediately before my arrival ; and the placenta followed, with slight assistance, shortly after. Nothing unusual had been noticed during labour, by patient or nurse, either in the pains or otherwise; and the uterus contracted well, with no hæmorrhage of consequence. Before long, however, respiration became hurried, with a pulse of 120 , small, and somewhat intermittent. A continuous pain, too, was complained of over the abdomen; also in the back, between the shoulders.

Stimulants were administered with no benefit, and it soon became evident that grave cause for anxiety existed. Presently, the pulse decreased still more in force; the usual signs of collapse setting in, except that the face and extremities were congested, instead of presenting the pallor which generally accompanies collapse. Towards midday, delirium, accompanied by coma, preceded the end.

A post mortem examination was made the following day, revealing a rupture of the vagina posteriorly, just below the cervix, and allowing blood to escape into the cavity of the abdomen. A generally unhealthy condition of organs existed, the heart being laden with fat on its surface, while the muscular tissue was exceedingly soft and friable. Both lungs were highly congested and full of serum, especially the right, which was closely adherent to the chest-wall.

On reference to authorities, rupture of the vagina alone* seems somewhat rare, and about half as fatal as that of the uterus. The symptoms are described as similar; but the collapse is less severe in vaginal rupture, with a probability of death, not so much from shock as from subsequent inflammation. In the present case; we may conclude that death was caused by rupture of the vagina; but the symptoms were complicated and the end accelerated by an unsound condition of the organs generally, and the heart in particular.

$$
\text { Walter Thomas Beeey, M.D., Bromley, Kent. }
$$

* I refer, of course, to lesions of the upper portions of the vagina, such as would communicate with the cavity of the abdomen and peritoneum. 